\title{
Magnetic Properties of $\mathrm{KGd}\left(\mathrm{WO}_{4}\right)_{2}$ Single Crystal Studied by EPR Spectroscopy
}

\author{
S. M. Kaczmarek ${ }^{1}$, G. Leniec ${ }^{1}$, T. Bodziony ${ }^{1}$, H. Fuks ${ }^{1}$, T. Skibiński ${ }^{1}$, J. Hanuza ${ }^{2}$ \& L. Macalik ${ }^{2}$ \\ ${ }^{1}$ Institute of Physics, West Pomeranian University of Technology, Szczecin, Poland \\ ${ }^{2}$ Institute of Low Temperature and Structure Research, PAS, Okólna, Wrocław, Poland \\ Correspondence: Sławomir M. Kaczmarek, Institute of Physics, Department of Mechanical Engineering and \\ Mechatronics, Szczecin, Poland. Tel: 48-91-449-4887. E-mail: skaczmarek@zut.edu.pl
}

Received: February 26, 2013 Accepted: March 23, 2013 Online Published: April 15, 2013

doi:10.5539/jmsr.v2n3p23 URL: http://dx.doi.org/10.5539/jmsr.v2n3p23

\begin{abstract}
Magnetic properties of magnetically concentrated $\mathrm{KGd}\left(\mathrm{WO}_{4}\right)_{2}$ single crystal are studied using EPR spectroscopy. The EPR spectra are broad and have a complex shape, showing contributions of a few gadolinium centers. Temperature dependence of the total intensity of the EPR spectra and the inverse of the total intensity reveal two temperatures when the behavior of the total intensity suddenly changes, first one at $66.8 \pm 3.3 \mathrm{~K}$ and the second one at $9.9 \pm 2.8 \mathrm{~K}$. Detailed analysis of this dependence suggests the presence of different magnetic systems of the $\mathrm{Gd}^{3+}$ ions. The EPR spectra were described by the spin Hamiltonian of monoclinic symmetry with electron spin $S=7 / 2$, for which all $g$-matrix components and fine-structure parameters have been determined using EPR-NMR program. Raman spectra measured at low temperatures do not differ significantly from the one measured at room temperature. The analysis has given a deeper insight into different kinds of magnetic interactions between paramagnetic $\mathrm{Gd}^{3+}$ centers in $\mathrm{KGd}\left(\mathrm{WO}_{4}\right)_{2}$ single crystal.
\end{abstract}

Keywords: double tungstate, $\mathrm{KGd}\left(\mathrm{WO}_{4}\right)_{2}$, EPR spectra, magnetic systems

\section{Introduction}

Single crystals of double tungstate $\mathrm{KGd}\left(\mathrm{WO}_{4}\right)_{2}$ (hereafter: $\mathrm{KGW}$ ) are receiving increasing attention as new and perspective materials for solid state lasers, especially in the development of the solid-state Raman laser (Adryunas et al., 1985). The monoclinic KGW crystal is a good laser host, providing high efficiency for stimulated emission at low pumping energies, both with lamp and laser diode excitation (Kaminskii, 1991; Kaminskii, Verdun, Koechner, \& Pavlyuk, 1992). Special attention attracts KGW single crystals doped with rare-earth elements like neodymium $\left(\mathrm{Nd}^{3+}\right)$ (Grabtchikov et al., 2001) or erbium $\left(\mathrm{Er}^{3+}\right)$ (Pujol et al., 1999). Doped with lanthanide ions KGW crystals could be applied in long-distance optical communicators (Pujol, Aguilo, Diaz, \& Zaldo, 1999). Both crystals belong to magnetically concentrated media.

The crystal structure of $\mathrm{KGd}\left(\mathrm{WO}_{4}\right)_{2}$ is monoclinic with space group $\mathrm{C} 2 / \mathrm{c}$. Yttrium lattice ions substitute for gadolinium ions. The lattice parameters of the unit cell are $a=10.652(4), b=10.374(6), c=7.582(2) \AA, \beta=$ $130.80(2)^{\circ}$ and $Z=4$ according Pujol et al. (Pujol et al., 2001; Macalik, Hanuza, \& Kaminskii, 2002). W and O atoms form $\mathrm{WO}_{4}{ }^{2-}$ octahedral units with sharing edges. These octahedra linking by common vertices constitute a double chain extended along the $c$ axis (Pujol et al., 1999). The $\mathrm{K}^{+}$and $\mathrm{Gd}^{3+}$ cations occupy $C_{2}$ point symmetry positions. Rare earth ions $\left(\mathrm{RE}^{3+}\right)$ doped the KGW crystal occupy the same positions as $\mathrm{Gd}^{3+}$. Tungsten atoms are connected via oxygen bridges. The vibrations of the single $\mathrm{W}^{\mathrm{O}} \mathrm{W}$ and double $\mathrm{W}^{\mathrm{O}}{ }_{\mathrm{O}} \mathrm{W}$ oxygen bridges play an important role in the spontaneous Raman scattering (SRS) (Adryunas et al., 1985; Macalik et al., 2002; Fuks et al., 2010; Macalik, Hanuza, \& Kaminskii, 2000) and are very important in possible application of this crystal as a Raman crystal (Adryunas et al., 1985).

Previously Cherney et al. (Cherney, Nadolinny, \& Pavlyuk, 2008) have calculated spin Hamiltonian parameters for $\mathrm{KY}\left(\mathrm{WO}_{4}\right)_{2}: \mathrm{Gd}^{3+}$ (10 to $10000 \mathrm{ppm}$ of $\mathrm{Gd}_{2} \mathrm{O}_{3}$ ) diluted system. They simulated experimental EPR spectra with using special programs and obtained $g_{x}=1.99 \pm 0.02, g_{y}=1.99 \pm 0.02$ and $g_{z}=2.00 \pm 0.01$. EPR spectrum of diluted double tungstate doped with gadolinium reveals clear fine structure. Fine structure parameters are defined by the crystal field of the local oxygen surrounding $\left(\mathrm{D}=3 \mathrm{~B}_{2}{ }^{0}, \mathrm{E}=\mathrm{B}_{2}{ }^{2}\right)$. They have found $\mathrm{B}_{2}{ }^{0}=416 \pm 6 \mathrm{G}$ and $\mathrm{B}_{2}{ }^{2}=-180 \pm 10 \mathrm{G}$ (the order of the parameters is preserved also in a paper by Borowiec et al. (1998)) and the difference in the D parameter between different centres of $\mathrm{KY}\left(\mathrm{WO}_{4}\right)_{2}$ : $\mathrm{Gd}$ is defined by the difference in 
distances Y-O, that in this case changes from 0.2284 to $0.3877 \mathrm{~nm}$. The shorter distance Gd-O when substituting yttrium by gadolinium in $\mathrm{KY}\left(\mathrm{WO}_{4}\right)_{2}$ implies the larger value of $\mathrm{B}_{2}{ }^{0}$, while the wider variation of these distances favours a larger value of $\mathrm{B}_{2}{ }^{2}$, which characterizes the rhombicity. This is a way to distinguish different types of gadolinium paramagnetic centers, characterized also by different values of exchange parameters.

In the first approximation the paramagnetic Curie temperatures depend on parameters $\mathrm{B}_{2}{ }^{0}$ and $\mathrm{B}_{2}{ }^{2}$ only, so it is easy to find exchange parameter characterizing exchange interactions between gadolinium ions. Borowiec et al. have stated that the comparison of the data obtained by magnetization measurements with those obtained in EPR measurements is justified because the diluted $\mathrm{KY}\left(\mathrm{WO}_{4}\right)_{2}$ crystal is isomorphic with $\mathrm{KGd}\left(\mathrm{WO}_{4}\right)_{2}$, so crystal fields acting on $\mathrm{Gd}^{3+}$ in $\mathrm{KY}\left(\mathrm{WO}_{4}\right)_{2}$ and in $\mathrm{KGd}\left(\mathrm{WO}_{4}\right)_{2}$ are expected to be similar. The supposition is true nevertheless, only for the first approximation, when one can neglect higher order terms in the spin Hamiltonian. Generally exchange and magnetic dipole-dipole interactions are comparable, so energies of all magnetic structures should be estimated to find the type of magnetic structure.

In this paper we analyze optical and magnetic properties of concentrated $\mathrm{KGd}\left(\mathrm{WO}_{4}\right)_{2}$ single crystal applying measurements and discussion of Raman and EPR spectra. Special attention is paid to EPR spectra analysis, as being very sensitive tool to describe magnetic properties of the investigated crystal, even it is a concentrated magnetic system.

\section{Experiment}

KGW single crystals were grown by a low-gradient Czochralski technique (modified top-seeded solution method) in Pt crucibles using oriented seeds along the crystallographic $\boldsymbol{b}$ axis. The crystals obtained were colourless and had high optical quality. Orientation of the crystals was done by X-ray method giving $\boldsymbol{a}, \boldsymbol{b}$ and $\boldsymbol{c}$ crystallographic axes (CAS) directions. $\boldsymbol{a}$ and $\boldsymbol{b}$ axes were the same for CAS axes system and experimental one (EAS), while $\boldsymbol{c}^{*}$ experimental axis was perpendicular to $\boldsymbol{a} \boldsymbol{b}$ plane (Macalik et al., 2002).

EPR spectra were recorded on a conventional X-band Bruker ELEXSYS E $500 \mathrm{CW}$-spectrometer operating at 9.5 GHz with $100 \mathrm{kHz}$ magnetic field modulation. Temperature dependence of the EPR spectra in "a" crystallographic direction ( $\boldsymbol{b} \boldsymbol{c}^{*}$-plane), from $6 \mathrm{~K}$ up to room temperature, was recorded using an Oxford Instruments ESP nitrogen-flow cryostat. The crystal was rotated around $\boldsymbol{a}, \boldsymbol{b}$ - and $\boldsymbol{c}^{*}$ - EAS at temperatures 7.5-11 $\mathrm{K}$, allowing draw angular dependences of EPR spectra. EPR-NMR program was applied to fit the dependences.

Raman spectra were measured in 4-300 K temperature range, using a Bruker RFS 100/S Raman Spectrometer with the back scattering configuration. The $1064 \mathrm{~nm}$ line of Nd:YAG laser was used as an excitation. Signal detection was performed with the LN-Ge (D418-T) liquid nitrogen-cooled NIR detector with an integrated preamplifier and high voltage power supply. The resolution of Raman spectra was $2 \mathrm{~cm}^{-1}$.

\section{Results and Discussion}

\subsection{Raman Spectra}

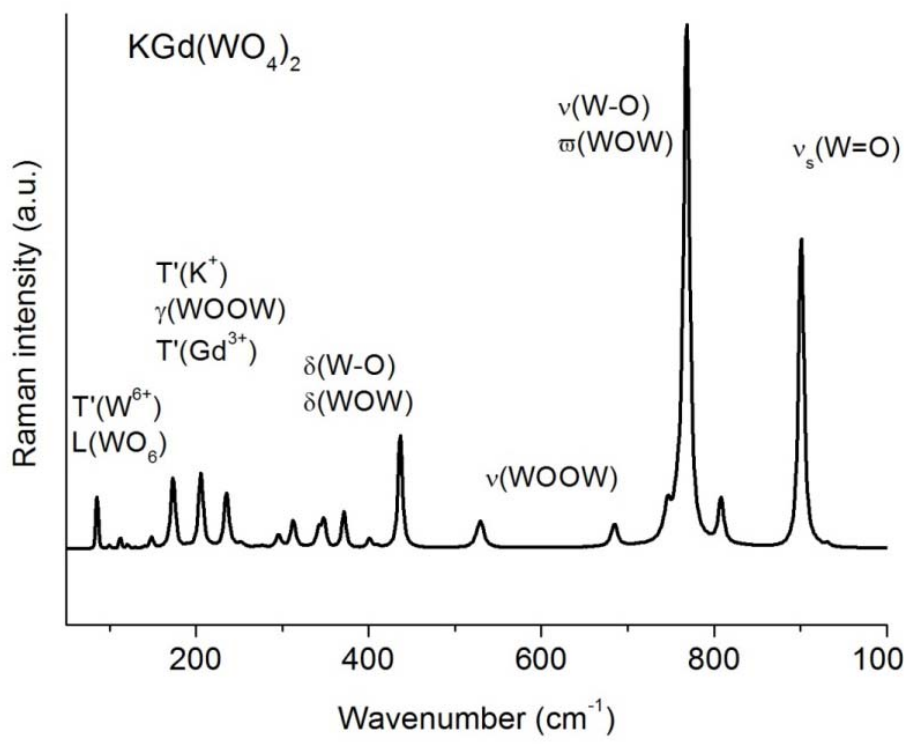

Figure 1. Raman spectrum of $\mathrm{KGd}\left(\mathrm{WO}_{4}\right)_{2}$ single crystal measured at room temperature 
The spectra measured in 4-200 K range (not presented in the Figure) do not differ significantly from the ones measured at room temperature (Figure 1). So we have not found any evidence on structure changes of the KGW crystal along the temperature range.

The Raman spectrum presented in Figure 1 consists of four strong bands in the 740-1000 $\mathrm{cm}^{-1}$ range (multiplets of the stretching $v(\mathrm{~W}-\mathrm{O})$ vibrations), two medium intensity bands in the $500-700 \mathrm{~cm}^{-1}$ range (stretching modes of the $\mathrm{W}^{\mathrm{O}} \mathrm{W}$ and $\mathrm{W}^{\mathrm{O}}{ }_{\mathrm{O}} \mathrm{W}$ oxygen bonds), medium intensity bands in the $270-470 \mathrm{~cm}^{-1}$ range (banding vibrations) and group of medium bands in the $70-260 \mathrm{~cm}^{-1}$ range (translational motions of the cations and anions, as well as vibrations of the tungstate polyhedra). The assignment to the respective normal modes presented in Figure 1 was done according to the previous works (Macalik et al., 2000; Pilbrow, 1990; Macalik et al., 1998). As compare to double tungstates built of isolated tetrahedra, $\mathrm{KGd}\left(\mathrm{WO}_{4}\right)_{2}$ polymeric crystal does not exhibit the energy gap between the 450 and $750 \mathrm{~cm}^{-1}$.

\subsection{EPR Measurements}

Figure 2 shows several EPR spectra (first derivative of the absorption) of KGW single crystal recorded at different temperatures. All the spectra were recorded at the same angle, when magnetic filed $(\boldsymbol{B})$ lied in the $\boldsymbol{b c}$-crystallographic plane. The temperature varied from $244 \mathrm{~K}$ (top line) down to $6 \mathrm{~K}$ (bottom line). One can notice that the EPR lines are very broad and have a complex shape. We have fitted them applying decomposition to at least three lines. This may suggest that the broad EPR signal registered for the KGW single crystal is a superposition of several signals from the same paramagnetic center but in a slightly different orientation in the crystal. So, the EPR spectra seem to be a superposition of a few signals originating from differently oriented $\mathrm{Gd}^{3+}$ centers. Changes in the shape, width and height of the EPR resonance lines are clearly visible when temperature decreases.

As one can see from Figure 2 the EPR spectra are typical rather for powders than for isolated $\mathrm{Gd}^{3+}$ centers. The total intensity of EPR spectrum is calculated by integration of the EPR absorption spectrum or as a double integral of the EPR spectrum (EPR susceptibility, $\chi_{E P R}$ ) (Pilbrow, 1990). Temperature dependence of the total intensity of the EPR spectra is presented in Figure 3 (upper panel, open squares). Solid line indicates Curie-Weiss law. The inverse of the total intensity is also shown in the same figure (lower panel, open circles). As one can see from Figure 3, the total intensity increases when temperature decreases. Curie-Weiss temperature, $\mathrm{T}_{\mathrm{c}}$, equals to $-118.34 \mathrm{~K}$, what indicates strong antiferromagnetic interactions between gadolinium ions. It is a typical behavior. But the total intensity changes considerably at a temperature about $70 \mathrm{~K}$, giving Curie-Weiss temperature $T_{c}=-21.39 \mathrm{~K}$. Very close behavior we reported previously for not oriented $\mathrm{KGW}$ single crystal (Fuks et al., 2010). It means impairment of gadolinium antiferromagnetic interactions. Next, the total intensity continues to increase until the temperature of $9.9 \mathrm{~K}$ is reached, when it starts to drop rapidly. The inverse of the total intensity behaves similarly. In this case we can recognize three ranges of linear change: first one up to $9.9 \mathrm{~K}$, the second one from 9.9 to $70 \mathrm{~K}$, and the third one from $70 \mathrm{~K}$ to $250 \mathrm{~K}$ (see Figure 3). We have got two temperatures, when the behavior of the total intensity considerably changes, first one at $66.8 \pm 3.3 \mathrm{~K}$ and the second one at $9.9 \pm 2.8 \mathrm{~K}$.

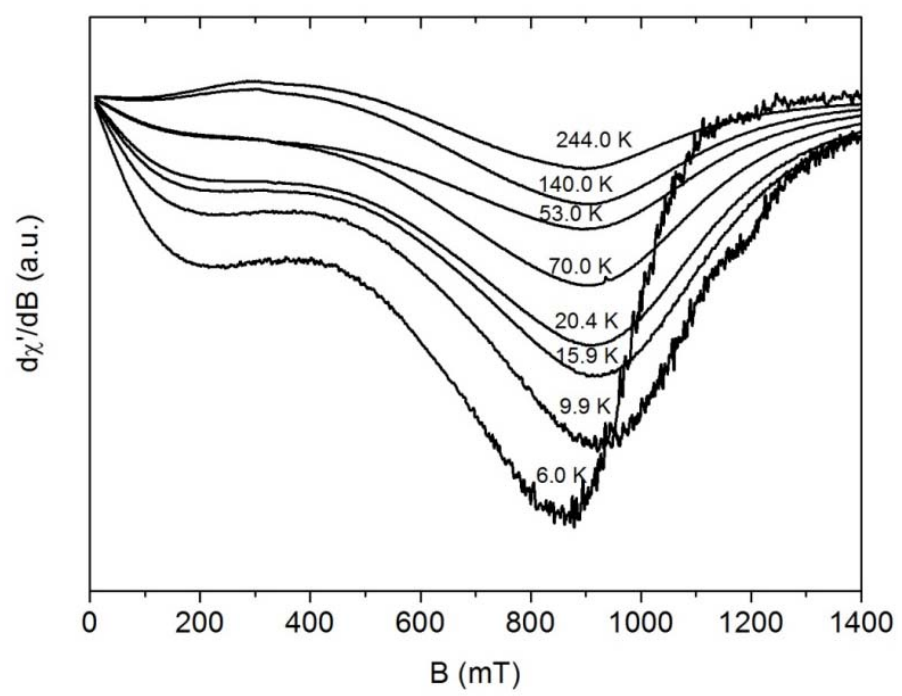

Figure 2. EPR spectra of $\mathrm{KGd}\left(\mathrm{WO}_{4}\right)_{2}$ single crystal recorded at eight different temperatures 


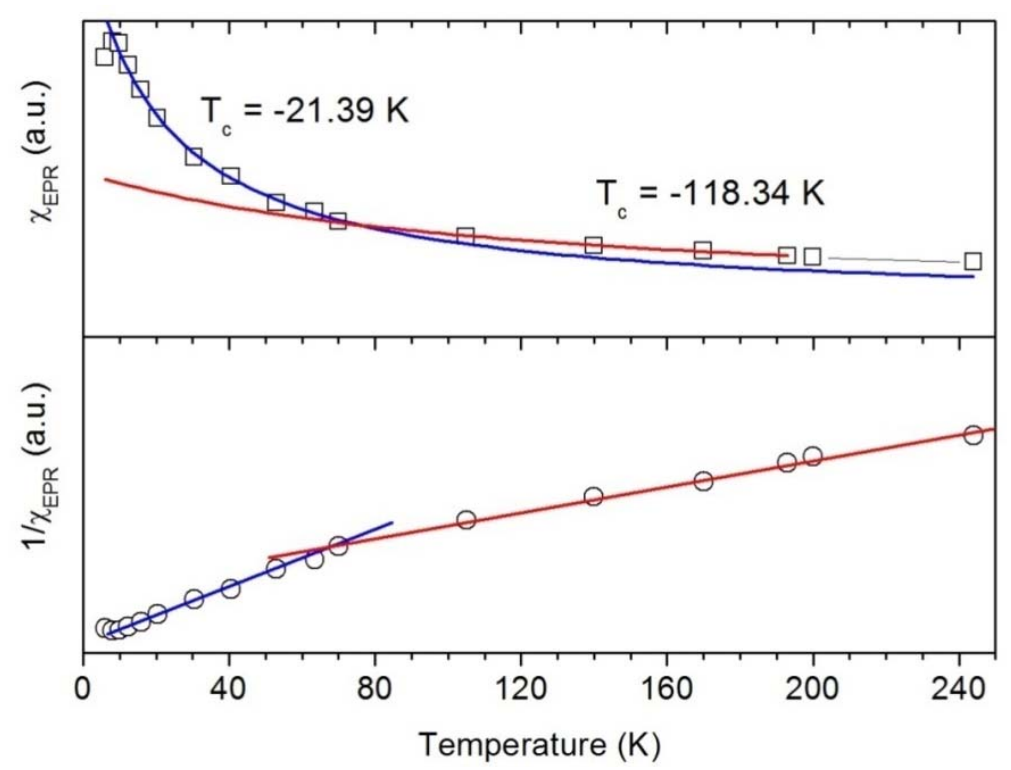

Figure 3. Temperature dependence of the total intensity (upper panel, open squares) and the inverse of the total intensity (lower panel, open circles) of EPR spectra for $\mathrm{KGd}\left(\mathrm{WO}_{4}\right)_{2}$ single crystal; $\boldsymbol{b} \boldsymbol{c}$ plane

Very crucial is dependence of spectroscopic $g$-factor versus temperature. When temperature increases from $6 \mathrm{~K}$ up to $10 \mathrm{~K}$ it suddenly decreases from 2.15 to 2.01 reaching minimum, next it increases reaching $g=2.08$ at 70 $\mathrm{K}$ (see inset in Figure 4). The dependence of a line-width, $\Delta B$, v.s. temperature reveals very similar complex behavior. We plotted it in Figure 4. As one can see in the temperature range where $g$ decreases, $\Delta B$ increases reaching maximum at about $19 \mathrm{~K}$ and next again decreases, presenting usual shape, characteristic of fast spin-lattice relaxation processes (short relaxation times). These dependences of $g$ and $\Delta B$ suggest the presence of structural or magnetic phase transition in the range between 6 and $19 \mathrm{~K}$ or strong change in magnetic ordering between gadolinium ions. Taking into account Raman spectra measurements described in section 3 of the paper the first supposition is not true.

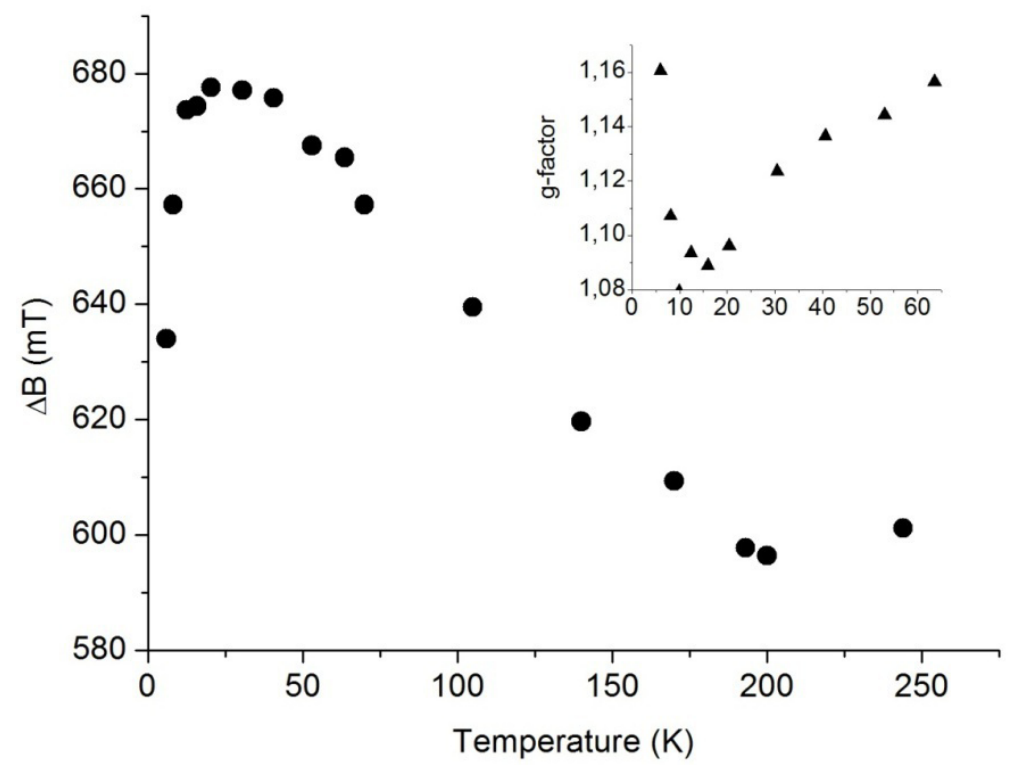

Figure 4. Line-width of the EPR spectrum versus temperature; inset shows g-factor dependence versus temperature in the $6-60 \mathrm{~K}$ range 
To analyze local symmetry of gadolinium ions centers, and, confirm their multiplicity inside $\mathrm{KGd}\left(\mathrm{WO}_{4}\right)_{2}$ single crystal suggested by EPR line decomposition, we have measured angular dependencies of the EPR resonance fields in all of three directions: $\boldsymbol{a}, \boldsymbol{b}$ and $\boldsymbol{c}^{*}$. They are presented in Figures $5 \mathrm{a}, \mathrm{b}$ and $\mathrm{c}$.

(a)

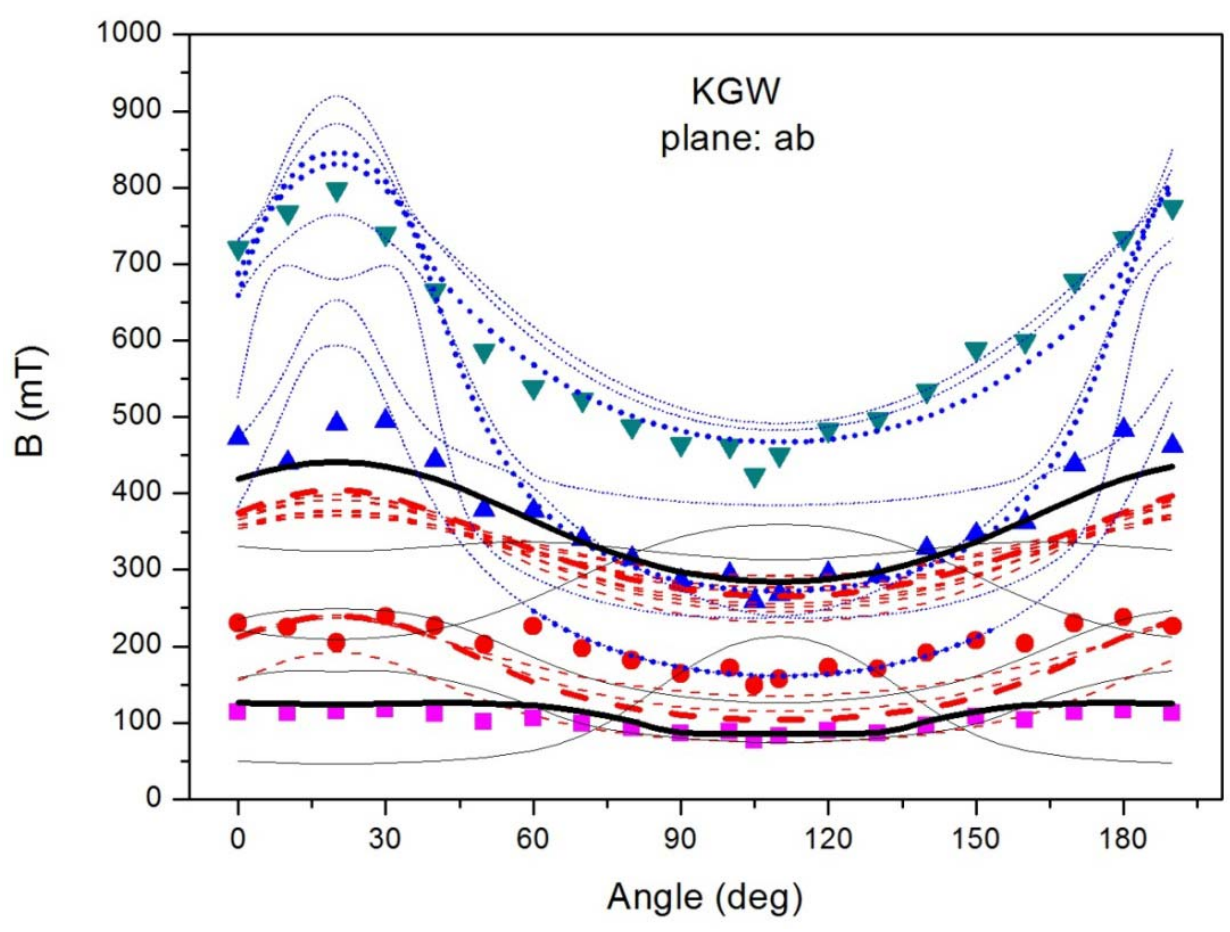

(b)

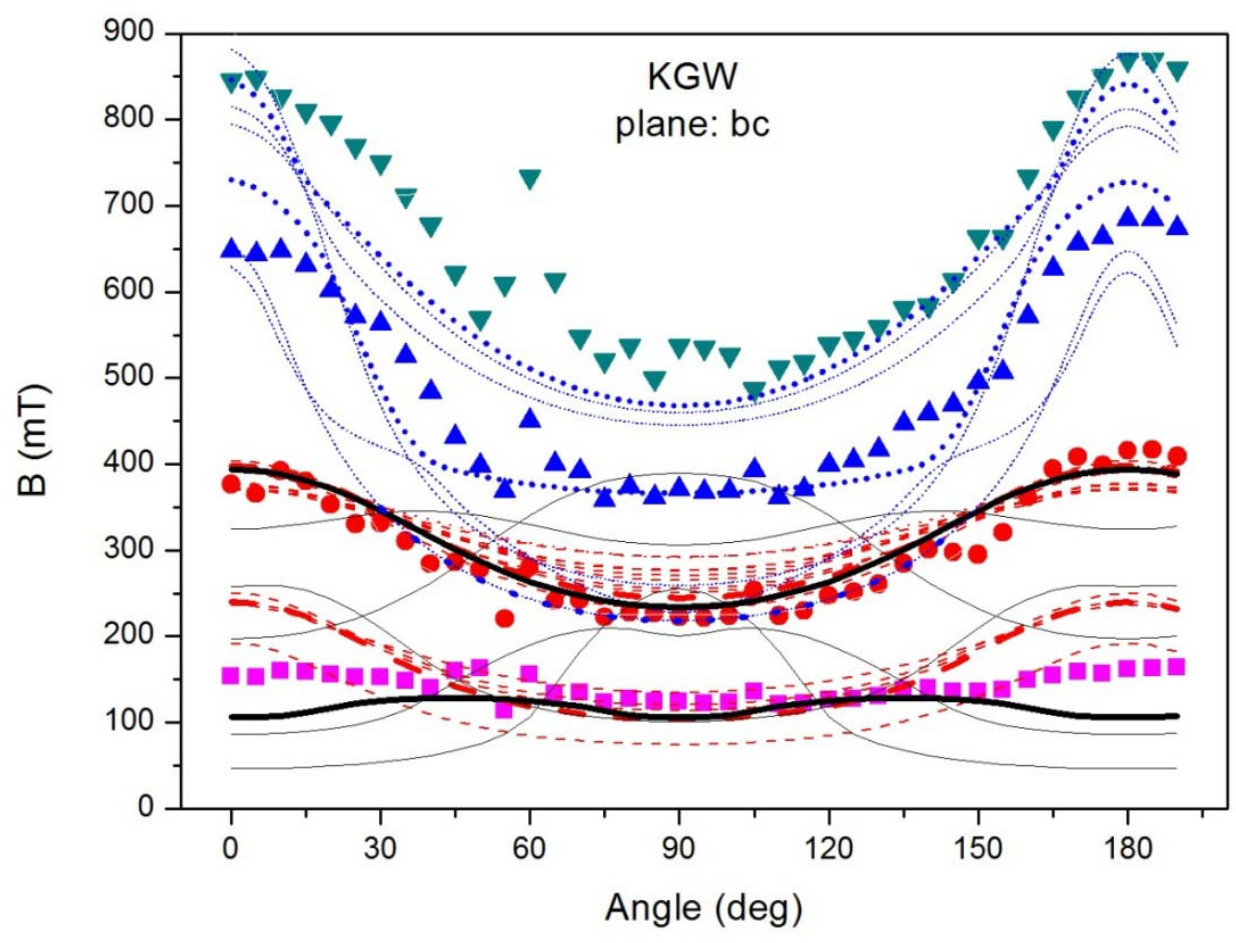


(c)

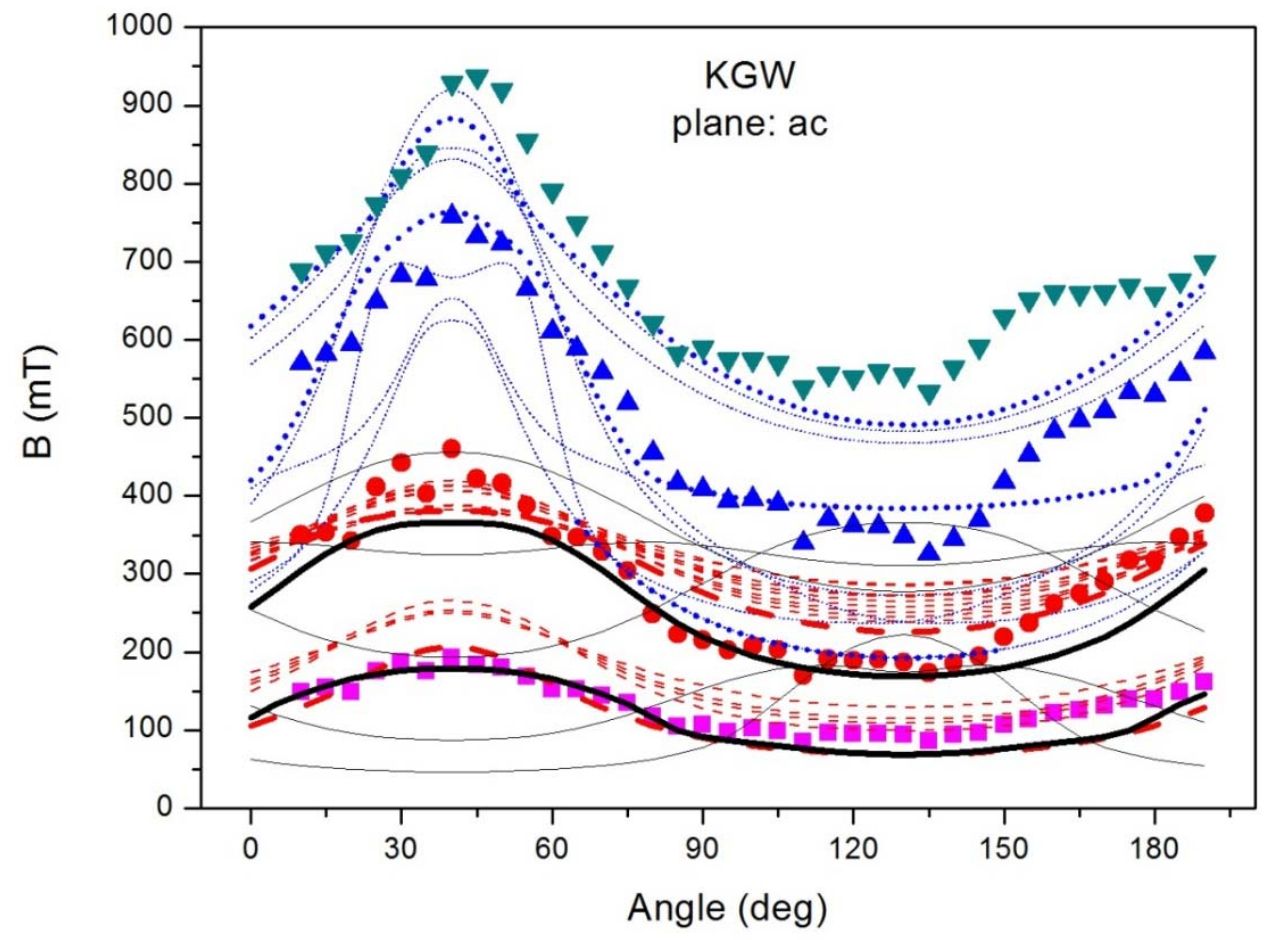

Figure 5. Angular dependences of EPR lines position for: (a) $\boldsymbol{a} \boldsymbol{b}$-plane at $10.5 \mathrm{~K}$, (b) $\boldsymbol{b} \boldsymbol{c}$-plane at $11 \mathrm{~K}$, (c) ac-plane at $7.5 \mathrm{~K}$; solid lines mark fitted lines, while dotted lines represent possible transition ( $\mathrm{S}=7 / 2)$ obtained from EPR-NMR program (roadmap)

As one can see from Figure 5 four lines (experimental points) are present in each of the analyzed crystal orientation. The lines reflect fine-structure of the Gd EPR spectra. Typically, the fine-structure of the Gd signal is well resolved into seven separated EPR lines originated from isolated gadolinium ions. The shape of angular dependences indicates on low symmetry of responsible magnetic centres, possible $C_{2}$ symmetry, as is expected for $\mathrm{Gd}^{3+}$ ions in double tungstate crystal structure.

The following spin Hamiltonian was used to generate angular dependence (roadmap) of the KGW crystal:

$$
\begin{gathered}
H=H_{\text {isolated }}+H_{\text {pair }} \\
H_{\text {isolated }}=g_{o} \mu_{B} B \cdot S+\sum_{m=-l}^{l} B_{l}^{m} \cdot O_{l}^{m}(S) \\
H_{\text {pair }}=g_{o} \mu_{B} B \cdot S_{1,2}+J S_{1} \cdot S_{2}+\sum_{m=-l}^{l} B_{l}^{m} \cdot O_{l}^{m}\left(S_{1,2}\right)
\end{gathered}
$$

where the first term is the electronic Zeeman term, the second one reflects pair interaction and third one contains the higher order $(l=2,4,6)$ Stevens parameters (crystal field). The symbols have got their usual meaning.

The roadmap was generated using EPR-NMR program (Mombourquette, Weil, \& McGavin, 1999). The results of the fitting are presented in Figures 5 as thin and bold lines, solid, dashed and dotted. The bold (solid, dashed and dotted) lines represent lines fitted to the experimental data, obtained using the Hamiltonian (1), while the thin lines (solid, dashed and dotted) have been generated for this match and represent invisible, but possible transitions attributed to gadolinium ions in host lattice. To extract Hamiltonian parameters, there were taken into account three diffident paramagnetic centers: isolated gadolinium ions (solid lines), gadolinium pairs with a weak interaction (dashed lines) and gadolinium pairs with a strong interaction (dotted lines). The number of the centers results from EPR line decomposition (at least three lines) and from fitting procedure. The EPR signal originated from the isolated gadolinium ions is well resolved between 50-500 $\mathrm{mT}$ of magnetic field (solid lines). But itis not enough to explain all of our results. Since the EPR signal in the above range is also broad one, we assigned the signal to weakly interacting pairs of gadolinium ions (dashed lines). Very similar signal is observed also for high magnetic fields, which we assigned to strongly interacting pairs of gadolinium ions (dotted lines). 
As can be seen from Figures 5, simulated lines describe experimental data well enough. At least two of the lines in each of planes were obtained for the same Hamiltonian parameters. Superposition of an EPR signal originating from many of the same paramagnetic ions but slightly shifted in the crystal lattice seem to be a reason why do some transitions are not observed. Hamiltonian parameters, obtained from the fitting procedure, are comparable to those, previously known in the literature (Table 1), (Fuks et al., 2010; Cherney et al., 2008). Nevertheless, some of them slightly differ from the standard values. One of possible explanation is coexistence of several kinds of gadolinium centers. The values of spin Hamiltonian parameters are gathered in Table 1a, b.

Table 1. The spin Hamiltonian parameters of $\mathrm{KGd}\left(\mathrm{WO}_{4}\right)_{2}$ single crystal

a).

\begin{tabular}{c}
\hline Gd- isolated centers \\
\hline$g=\left[\begin{array}{lll}2.08 & \\
& 2.07 & \\
& 2.14\end{array}\right]$ \\
\hline$B_{2}^{0}=268(10) \cdot 10^{-4} \mathrm{~cm}^{-1}$ \\
$B_{2}^{2}=-135(10) \cdot 10^{-4} \mathrm{~cm}^{-1}$ \\
\hline$B_{4}^{0}=1.8(2) \cdot 10^{-4} \mathrm{~cm}^{-1}$ \\
$B_{4}^{2}=1.6(2) \cdot 10^{-4} \mathrm{~cm}^{-1}$ \\
$B_{4}^{4}=1.5(2) \cdot 10^{-4} \mathrm{~cm}^{-1}$ \\
$B_{4}^{-2}=2.0(2) \cdot 10^{-4} \mathrm{~cm}^{-1}$ \\
\hline$B_{6}^{0}=-1.5(5) \cdot 10^{-4} \mathrm{~cm}^{-1}$ \\
$B_{6}^{2}=-1.5(2) \cdot 10^{-4} \mathrm{~cm}^{-1}$ \\
$B_{6}^{4}=0.3(1) \cdot 10^{-4} \mathrm{~cm}^{-1}$ \\
$B_{6}^{-2}=0.1(1) \cdot 10^{-4} \mathrm{~cm}^{-1}$ \\
$B_{6}^{-4}=2.0(2) \cdot 10^{-4} \mathrm{~cm}^{-1}$
\end{tabular}

b).

\begin{tabular}{cc}
\hline Gd-Gd $\quad$ - dashed lines in Figures 5 & Gd-Gd - dotted lines in Figures 5 \\
\hline$g=\left[\begin{array}{lll}1.99 & & \\
& 1.99 & \\
& & 2.01\end{array}\right]$ & $g=\left[\begin{array}{ll}1.86 & \\
& 1.89 \\
& \\
\hline B_{2}^{0}=285(8) \cdot 10^{-4} \mathrm{~cm}^{-1}\end{array}\right]$ \\
$B_{2}^{2}=-100(10) \cdot 10^{-4} \mathrm{~cm}^{-1}$ & $B_{2}^{0}=275(8) \cdot 10^{-4} \mathrm{~cm}^{-1}$ \\
\hline$B_{4}^{0}=1.8(2) \cdot 10^{-4} \mathrm{~cm}^{-1}$ & $B_{2}^{2}=-100(10) \cdot 10^{-4} \mathrm{~cm}^{-1}$ \\
$B_{4}^{2}=1.6(2) \cdot 10^{-4} \mathrm{~cm}^{-1}$ & $B_{4}^{0}=1.8(2) \cdot 10^{-4} \mathrm{~cm}^{-1}$ \\
$B_{4}^{4}=1.5(2) \cdot 10^{-4} \mathrm{~cm}^{-1}$ & $B_{4}^{2}=1.6(2) \cdot 10^{-4} \mathrm{~cm}^{-1}$ \\
$B_{4}^{-2}=2.0(2) \cdot 10^{-4} \mathrm{~cm}^{-1}$ & $B_{4}^{4}=1.5(2) \cdot 10^{-4} \mathrm{~cm}^{-1}$ \\
\hline$B_{6}^{0}=-1.45(5) \cdot 10^{-4} \mathrm{~cm}^{-1}$ & $B_{4}^{-2}=2.0(2) \cdot 10^{-4} \mathrm{~cm}^{-1}$ \\
$B_{6}^{2}=-1.5(2) \cdot 10^{-4} \mathrm{~cm}^{-1}$ & $B_{6}^{0}=-1.45(5) \cdot 10^{-4} \mathrm{~cm}^{-1}$ \\
$B_{6}^{4}=0.3(1) \cdot 10^{-4} \mathrm{~cm}^{-1}$ & $B_{6}^{2}=-1.5(2) \cdot 10^{-4} \mathrm{~cm}^{-1}$ \\
$B_{6}^{-2}=0.1(1) \cdot 10^{-4} \mathrm{~cm}^{-1}$ & $B_{6}^{4}=0.3(1) \cdot 10^{-4} \mathrm{~cm}^{-1}$ \\
$B_{6}^{-4}=2.0(2) \cdot 10^{-4} \mathrm{~cm}^{-1}$ & $B_{6}^{-2}=0.1(1) \cdot 10^{-4} \mathrm{~cm}^{-1}$ \\
\hline $\mathrm{J}_{\mathrm{diag}}=-70(10) \cdot 10^{-4} \mathrm{~cm}^{-1}$ & $B_{6}^{-4}=2.0(2) \cdot 10^{-4} \mathrm{~cm}^{-1}$ \\
\hline
\end{tabular}

Elements of $g$ and $B_{l}^{m}$ matrices calculated using EPR-NMR program for $\mathrm{KGd}\left(\mathrm{WO}_{4}\right)_{2}$ single crystal: a) for isolated gadolinium centers and b) for two kinds of pairs of gadolinium ions; Stevens parameters are given in Gauss units, $J$-exchange constant. 


\section{Discussion}

To discuss our results we need a deeper insight into the structure of the unit cell of KGW single crystal. Double tungstates are characterized by the double chain along the crystallographic $c$ direction of two $\mathrm{W}_{2} \mathrm{O}_{8}$ distorted octahedra sharing $\mathrm{O}-\mathrm{O}$ edges. The coordination figure of the tungstate anion is a distorted octahedron $\mathrm{WO}_{6}$. $\mathrm{RE}^{3+}$ ions are eight-coordinated by $\mathrm{O}$ atoms, forming a square antiprism. These polyhedra form a single zig-zag chain in the direction (101) by sharing edges. The alkali cation $\mathrm{K}^{+}$is twelve- coordinated by $\mathrm{O}$ ions, forming distorted icosahedrons (Pujol et al., 2001). A projection of $\mathrm{KGd}\left(\mathrm{WO}_{4}\right)_{2}$ crystalline structure, along the $\boldsymbol{b}$ axis, is shown in Figure 6. The positions of $\mathrm{Gd}^{3+}$ and $\mathrm{K}^{+}$ions and chains of connected $\mathrm{WO}_{6}$ octahedra are well visible. The resonance signal originates from $\mathrm{Gd}^{3+}(\mathrm{S}-$ state ions $-\mathrm{L}=0$ ) (Pilbrow, 1990; Borowiec et al., 2010). But resonance signals originating from isolated $\mathrm{Gd}^{3+}$ ions with well resolved seven lines are not visible in the EPR spectra of KGW single crystal (see Figure 2). Instead a few narrow lines we observe only one, very broad resonance line. Such EPR spectra are typical rather for powders than for isolated $\mathrm{Gd}^{3+}$ centers. It means that the lattice relaxation times of the paramagnetic centers are very short. It may suggest that $\mathrm{Gd}^{3+}$ ions in $\mathrm{KGW}$ crystal are strongly connected via magnetic interactions.

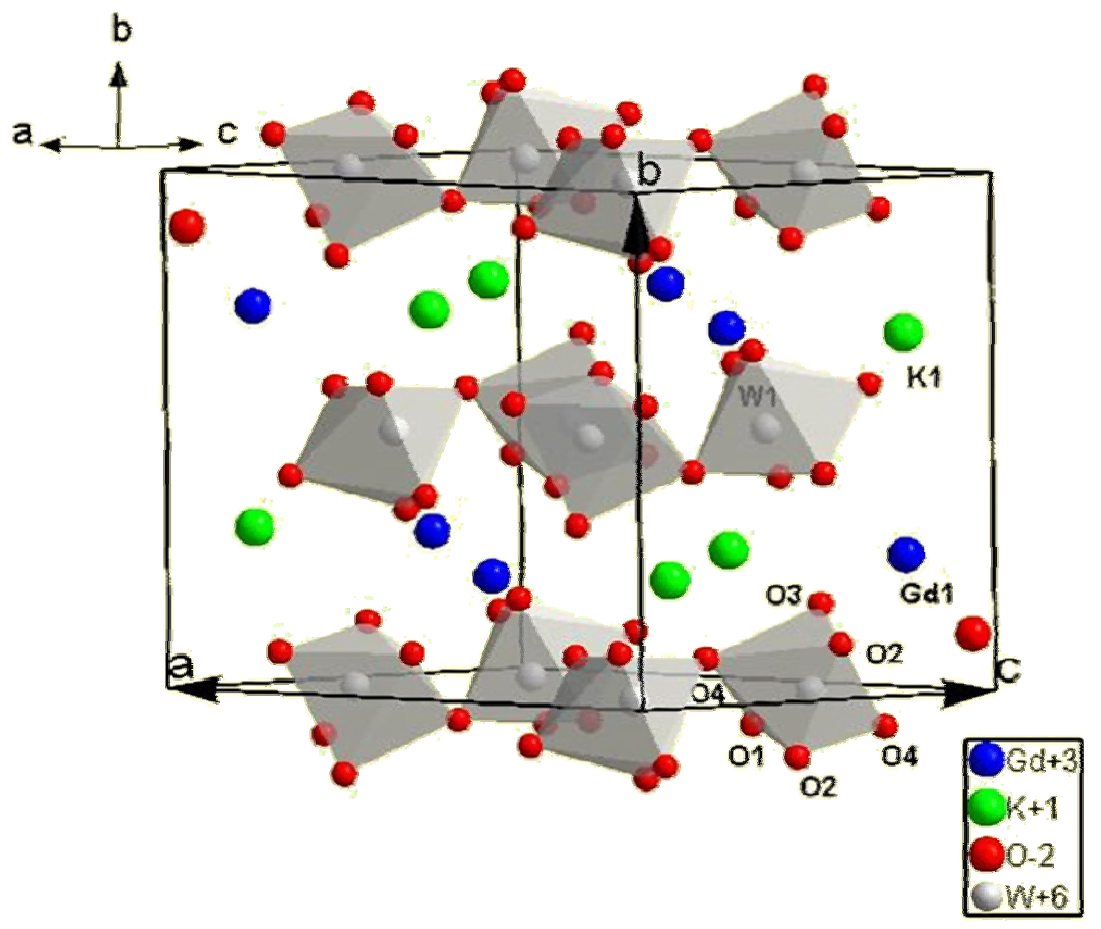

Figure 6. Projection of $\mathrm{KGd}\left(\mathrm{WO}_{4}\right)_{2}$ crystalline structure parallel to the $\boldsymbol{b}$ axis

We have used four Gaussian shape lines to fit EPR spectra. A superposition of these constituent lines very well describes the EPR spectra. The shape of the constituent lines indicates on dipole-dipole magnetic interactions. Thus, magnetically concentrated system such like $\mathrm{KGd}\left(\mathrm{WO}_{4}\right)_{2}$ shows strong dipole-dipole coupling which impedes the analysis of single-ion magnetic interaction effects. Moreover, this superposition may suggest that several magnetically different systems are present in the KGW single crystal. These magnetic systems may be assigned to different kinds of magnetic interactions between $\mathrm{Gd}^{3+}$ ions, especially pairs of ions. Individual systems may determine the behavior of the EPR spectra in different temperature and magnetic field ranges.

Temperature dependence of the total intensity of the EPR spectra and the inverse of the total intensity show two different "jumps" at temperatures: $66.8 \pm 3.3 \mathrm{~K}$ and $9.9 \pm 2.8 \mathrm{~K}$ (see Figure 3). In magnetic susceptibility measurements did by Borowiec et al. (2010) such "jumps" were not observed. For this crystal orientation, nevertheless, they presented experimental points of susceptibility measurements only up to $\sim 80 \mathrm{~K}$. Both "jumps" could be recognized in our investigations due to better accuracy of EPR experiment. Taking into account results of Raman spectra analysis, we could explain such behavior of temperature dependence of the total EPR intensity by assuming that at these specific temperatures a change in a kind of dominating magnetic interactions or activity of different magnetic systems takes place. We can deal, e.g., with inter-chains, intra-chains and pairs or 
clusters. Such conclusion confirm angular dependences of EPR spectra (see also Table 1b) and a shape of Raman spectrum (Figure 1), in which, between 450 and $750 \mathrm{~cm}^{-1}$ specific lines assigned to stretching modes of the $\mathrm{W}^{\mathrm{O}} \mathrm{W}$ and $\mathrm{W}_{0}^{\mathrm{O}}{ }_{\mathrm{O}} \mathrm{W}$ oxygen bonds are observed. $\mathrm{In} \mathrm{KGd}\left(\mathrm{WO}_{4}\right)_{2}$ tungstates, crystallizing in the monoclinic structure, the tungstate units built the $\mathrm{WO}_{6}$ octahedra joined through the single and double oxygen bridges, so $\mathrm{KGd}\left(\mathrm{WO}_{4}\right)_{2}$ has got polymeric structure, which is a source of complex magnetic behavior between gadolinium ions.

EPR spectra of $\mathrm{KGd}\left(\mathrm{WO}_{4}\right)_{2}$ single crystal and elements of its $\boldsymbol{g}$ matrix presented in Table 1a, a little bit differ from similar ones calculated for diluted medium e.g. in (Cherney et al., 2008). Nevertheless, two main spin Hamilitonian parameters, $\mathrm{B}_{2}{ }^{0}$ and $\mathrm{B}_{2}{ }^{2}$ (see Table 1), describing in the first approximation magnetic properties of $\mathrm{KGd}\left(\mathrm{WO}_{4}\right)_{2}$ single crystal, stay with good enough agreement with these calculated in (Cherney et al., 2008; Borowiec et al., 1998). Also exchange parameters, $J$, do not significantly differ from these calculated in (Borowiec et al., 2010). Regularity in a change of $\mathrm{B}_{2}{ }^{0}$ and $\mathrm{B}_{2}{ }^{2}$ spin Hamiltonian parameters described in (Cherney et al., 2008) and discussed by us in Introduction, is not fully preserved (see Table 1b, c). It may be an effect of magnetically concentrated medium and strong dipole-dipole interactions. What does result from the observed differences? First of all there is not clearly visible the fine-structure in the EPR spectrum of concentrated medium, whose parameters are defined by the crystal field of local oxygen surrounding. It was observed previously by Leniec et al. in (Leniec, Macalik, Kaczmarek, Skibiński, \& Hanuza, 2012). So, EPR spectra of concentrated and diluted media could not be compared immediately. In magnetically concentrated medium, moreover, like $\mathrm{KGd}\left(\mathrm{WO}_{4}\right)_{2}, \mathrm{KGd}\left(\mathrm{WO}_{4}\right)_{2}: \mathrm{Nd}$ or $\mathrm{KGd}\left(\mathrm{WO}_{4}\right)_{2}: \mathrm{Er}$ different $\mathrm{Gd}^{3+}$ centers may arise because there exist different Y-O distances. For concentrated medium one can expect full representation of Gd-O distances as compare to diluted one. So, paired centers could be more often represented in the concentrated lattice than in diluted one. The above mentioned discrepancy in values of $\boldsymbol{g}$ matrix between the two media may be also due to strong dipole-dipole interactions.

Moreover, gadolinium ions in the crystal may interact with each other by two ways: a) through space or b) through bonds (Bencini \& Gatteschi, 1990). The structure of the KGW unit cell indicates that both ways of $\mathrm{Gd}^{3+}$ ions interaction are possible. The distance between two nearest $\mathrm{Gd}^{3+}$ ions is equal to 4.0701(19) $\AA$ (Pujol et al., 2001). There are four $\mathrm{Gd}^{3+}$ ions in such distances forming a chain. The ions within a chain may easily interact. Similarly, $\mathrm{Gd}^{3+}$ ions interacting via $\mathrm{O}^{2-}$ oxygen bridges may form magnetically inequivalent structures, from pairs of $\mathrm{Gd}^{3+}-\mathrm{Gd}^{3+}$ ions to various clusters of $\mathrm{Gd}^{3+}$ ions. One can apply a law-dimensional Ising chain model to describe the behaviour of the total intensity of the EPR spectra (Borowiec et al., 2010).

\section{Conclusions}

The results of the investigations of the EPR spectra of $\mathrm{KGd}\left(\mathrm{WO}_{4}\right)_{2}$ single crystal recorded at different temperatures and crystallographic orientations can be summarized as follows:

- The Raman spectra measured in the $4-200 \mathrm{~K}$ range do not differ significantly from the one measured at room temperature; there were not found structure changes in $\mathrm{KGd}\left(\mathrm{WO}_{4}\right)_{2}$ single crystal under temperature variation;

- EPR measurements performed in a temperature range of 6-244 $\mathrm{K}$ show a broad resonance line with complex shape; EPR spectra have been fitted very well with four Gaussian shape lines;

- Temperature dependence of the total intensity of the EPR spectra and the inverse of the total intensity reveal two "jumps" at temperatures: $66.8 \pm 3.3 \mathrm{~K}$ and $9.9 \pm 2.8 \mathrm{~K}$; at these specific temperatures a change in a kind of dominating magnetic interactions or activity of different magnetic systems takes place;

- At least two lines were registered and fitted in angular dependences for all crystallographic directions: $\boldsymbol{a} \boldsymbol{b}, \boldsymbol{a c}$ and $\boldsymbol{b} \boldsymbol{c}$, giving spin Hamiltonian parameters;

- Detailed analysis of angular dependencies revealed the presence of magnetic systems forming centres with spin $\mathrm{S}=7 / 2$ in monoclinic $\left(\mathrm{C}_{2}\right)$ symmetry;

- At least three types of gadolinium paramagnetic centres we found to be present in concentrated $\mathrm{KGd}\left(\mathrm{WO}_{4}\right)_{2}$ system: isolated $\mathrm{Gd}$ centres and two kinds of gadolinium pairs differing by a strength of exchange interaction, defined by exchange constant $\left(70 \times 10^{-4} \mathrm{~cm}^{-1}\right.$ and $\left.590 \times 10^{-4} \mathrm{~cm}^{-1}\right)$, very close to calculated in (Borowiec et al., 2010).

\section{References}

Adryunas, K., Vishchakas, Yu., Kabelka, V., Mochalov, I. V., Pavlyuk, A. A., Petrovskii, G. T., \& Svrus, V. (1985). Stimulated-Raman self-conversion of $\mathrm{Nd}^{3+}$ laser light in double tungstenate crystals. Jounal of 
Experimental and Theoretical Physics Letters, 42, 410-412.

Bencini, A., \& Gatteschi, D. (1990). Electron Paramagnetic Resonance of Exchange Coupled Systems. Berlin: Springer.

Borowiec, M. T., Dyakonov, V., Kamenev, V., Krygin, I., Piechota, S., Prokhorov, A., \& Szymczak, H. (1998). Pressure Effect on the $\mathrm{Gd}^{3+}$ Ion Ground State in the $\mathrm{KY}\left(\mathrm{WO}_{4}\right)_{2}+0.5 \% \mathrm{Gd}$ Crystal. Physica Status Solidi (b), 209, 443-448. http://dx.doi.org/10.1002/(SICI)1521-3951(199810)209:2<443::AID-PSSB443>3.0.CO;2-T

Borowiec, M. T., Zayarnyuk, T., Pujol, M. C., Aguilo, M., Diaz, F., Prokhorov, A. D., ... Szymczak, H. (2010). Magnetic properties of $\mathrm{KRE}\left(\mathrm{WO}_{4}\right)_{2}(\mathrm{RE}=\mathrm{Gd}, \mathrm{Yb}, \mathrm{Tm})$ single crystals. Physica B, 405, 4886-4891. http://dx.doi.org/10.1016/j.physb.2010.09.02

Cherney, N., Nadolinny, V. A., \& Pavlyuk, A. A. (2008). ESR investigation of $\mathrm{Gd}^{3+}$ ion introduction into the structure of simple and double tungstates. Applied Magnetic Resonance, 33, 45-55. http://dx.doi.org/10.1007/s00723-008-0057-0

Fuks, H., Kaczmarek, S. M., Leniec, G., Macalik, L., Macalik, B., \& Hanuza, J. (2010). EPR and vibrational studies of some tungstates and molybdates single crystals. Optical Materials, 32, 1560-1567. http://dx.doi.org/10.1016/j.optmat.2010.06.018

Grabtchikov, A. S., Kuzmin, A. N., Lisinetskii, V. A., Orlovich, V. A., Demidovich, A. A., Yumashev, K. V., ... Danilov, M. B. (2001). Pasively Q-swiched $1.35 \mu \mathrm{m}$ diode pumped Nd:KGW laser with V:YAG saturable absorber. Optical Materials, 16, 349-352. http://dx.doi.org/10.1016/S0925-3467(00)00099-9

Kaminskii, A. A. (1991). Achievements of modern crystal-laser physics. Annals of Physics France, 16, 639-706. http://dx.doi.org/10.1051/anphys:01991001606063900

Kaminskii, A. A., Verdun, H. R., Koechner, W., \& Pavlyuk, F. A. (1992). Efficient single-mode CW lasers based on monoclinic double potassium-(rare-earth) tungstenate crystals containing $\mathrm{Nd}^{3+}$ ions with semiconductor-laser pumping. Soviet Journal of Quantum Electronics, 22(10), 875-877. http://dx.doi.org/10.1070/QE1992v022n10ABEH003622

Leniec, G., Macalik, L., Kaczmarek, S. M., Skibiński, T., \& Hanuza, J. (2012). EPR and optical properties of $\mathrm{KY}\left(\mathrm{WO}_{4}\right)_{2}: \mathrm{Gd}^{3+}$ powders. Journal of Materials Research, 27(23), 2973-2981. http://dx.doi.org/10.1557/jmr.2012.345

Macalik, L., Hanuza J., \& Kaminskii, J. J. (2002). Polarized infrared and Raman spectra of $\mathrm{KGd}\left(\mathrm{WO}_{4}\right)_{2}$ and their interpretation based on normal coordinate analysis. Journal of Raman Spectroscopy, 33, 92-103. http://dx.doi.org/10.1002/jrs.829.abs

Macalik, L., Hanuza, J., \& Kaminskii, A. A. (2000). Polarized Raman spectra of the oriented $\mathrm{NaY}\left(\mathrm{WO}_{4}\right)_{2}$ and $\mathrm{KY}\left(\mathrm{WO}_{4}\right)_{2}$ single crystals. Journal of Molecular Structure, 555, 289-297. http://dx.doi.org/10.1016/S0022-2860(00)00612-8

Macalik, L., Hanuza, J., Macalik, B., Ryba-Romanowski, W., Gołąb, S., \& Pietraszko, A. (1998). Optical spectroscopy of $\mathrm{Dy}^{3+}$ ions doped in $\mathrm{KY}\left(\mathrm{WO}_{4}\right)_{2}$ crystals. Journal of Luminescence, 79, 9-19. http://dx.doi.org/10.1016/S0022-2313(98)00020-9

Mombourquette, M. J., Weil, J. A., \& McGavin, D. G. (1999). EPR-NMR User's Manual. Department of Chemistry, University of Saskatchewan, Saskatoon, SK, Canada.

Pilbrow, J. R. (1990). Transition Ion Electron Paramagnetic Resonance. Oxford: Clarendon Press.

Pujol, M. C., Aguilo, M., Diaz, F., \& Zaldo, C. (1999). Growth and characterization of monoclinic $\mathrm{KGd}_{1-\mathbf{x}} \mathrm{RE}_{\mathbf{x}}\left(\mathrm{WO}_{4}\right)_{2} \quad$ single crystals. Optical Materials, $13, \quad 33-40$. http://dx.doi.org/10.1016/S0925-3467(99)00008-7

Pujol, M. C., Sole, R., Massons, J., Gavalda, J., Solans, X., Zoldo, C., ... Aguilo, M. (2001). Structural study of monoclinic $\mathrm{KGd}\left(\mathrm{WO}_{4}\right)_{2}$ and effects of lanthanide substitution. Journal of Applied Crystallography, 34, 1-6. http://dx.doi.org/10.1107/S0021889800013352

Pujol, M. C., Zoldo, C., Sole, R., Nikolov, V., Solans, X., Aguilo, M., \& Diaz, F. (1999). Crystalline structure and optical spectroscopy of $\mathrm{Er}^{3+}$-doped $\mathrm{KGd}\left(\mathrm{WO}_{4}\right)_{2}$ single crystals. Journal of Applied Physics B, 68, 187-197. http://dx.doi.org/10.1007/s003400050605 